\title{
Adenovirus-mediated expression of SSAT inhibits colorectal cancer cell growth in vitro
}

\author{
Hui SUN ${ }^{2}$, Bin LIU², Ya-pei YANG, Chun-xiao XU, Yun-fei YAN, Wei WANG, Xian-xi LIU³ \\ ${ }^{2}$ Institute of Biochemistry and Molecular Biology, School of Medicine, Shandong University, Jinan 250012, China
}

\author{
Key words \\ spermidine/spermine $\mathrm{N}^{1}$-acetyltransferase; \\ adenovirus; colorectal cancer \\ ${ }^{1}$ Project supported by the Shandong Province \\ Natural Science Great Item (No Z2004C01) \\ and the Doctoral Foundation of the Ministry \\ of Education (No 20040422029). \\ ${ }^{2}$ These two authors contributed equally to \\ this work. \\ ${ }^{3}$ Correspondence to Dr Xian-xi LIU. \\ Phn 86-531-8838-2346. \\ E-mail xianxi@sdu.edu.cn \\ Received 2007-10-12 \\ Accepted 2008-01-07 \\ doi: $10.1111 / \mathrm{j} .1745-7254.2008 .00779 . x$
}

\begin{abstract}
Aim: To construct a recombinant adenovirus that can express human spermidine/ spermine $\mathrm{N}^{1}$-acetyltransferase (SSAT) and detect its inhibitory effect on colorectal cancer cell growth in vitro. Methods: A $516 \mathrm{bp}$ cDNA of SSAT was amplified and cloned into a pGL3-hTERT plasmid. The pGL3-hTERT-SSAT recombinant was digested, and the small fragment was cloned into the shuttle vector pAdTrack. The pAdTrack-hTERT-SSAT plasmids were recombined with pAdEasy-1 vectors in AdEasy-1 cells. Positive clones were selected and transfected into the HEK293 packaging cells (transformed human embryonic kidney cells) after they were linearized by $P a c I$. The process of adenovirus packaging and amplification was monitored by green fluorescent protein (GFP) expression. The SSAT protein levels were determined by Western blotting, and the intracellular polyamine content was detected by reverse-phase high performance liquid chromatography. The MTS (3-(4, 5-dimethylthiaol-2-yl)-5-(3-carboxy-methoxyphenyl)-2-(-4sulfophenyl)-2H-tetrazolium, inner salt) and colony-forming assays were used to analyze the gene transduction efficiency and effect on the growth of HT-29 and LoVo cells. A viable cell count was used to determine the cell growth with or without exogenous polyamines. Results: The GFP expression in 293 cells during virus packing and amplification was observed by fluorescence microscopy. Western blotting results demonstrated that Ad-hTERT-SSAT could increase the expression of SSAT, and consequently, spermidine and spermine were reduced to low levels. The MTS and colony-forming assay results showed that HT-29 and LoVo cell growth were significantly inhibited, and the inhibitory effect could be partially reversed by exogenous spermidine and spermine. Conclusion: The successfully constructed recombinant adenovirus Ad-hTERT-SSAT could accelerate polyamine catabolism and inhibit the colorectal cell growth in vitro. It also has therapeutic potential in the treatment of colorectal cancer.
\end{abstract}

\section{Introduction}

The polyamines, spermidine, spermine, and the diamine precursor putrescine are positively-charged aliphatic amines at physiological conditions, with a low-molecular weight and a simple chemical structure. They interact with various macromolecules, both electrostatically and covalently, and as a consequence, have a variety of cellular effects. The polyamines are typically met by the integrated contributions of biosynthesis, catabolism, uptake, and export, each of which is sensitively regulated by effector molecules that in turn are controlled by intracellular polyamine pools ${ }^{[1]}$. Thus, ornithine decarboxylase (ODC) and S-adenosylmethionine decarboxylase (AdoMetDC) control biosynthesis, a polyamine transport system modulates uptake, and spermidine/spermine $\mathrm{N}^{1}$ acetyltransferase (SSAT) regulates polyamine catabolism and export of the cell.

The polyamines are known to be critically involved in cell growth and have been implicated in the process of cell transformation $^{[2,3]}$. The level of polyamines is high in cancer cells and tissues, and rapid tumor growth has been associated 
with remarkable elevation of polyamine accumulation ${ }^{[4,5]}$. High polyamine levels have been detected in most cancers ${ }^{[6-8]}$. In colorectal cancer, the polyamine content is increased 3-4-fold more than those found in the equivalent normal tissue ${ }^{[9,10]}$.

The depletion of intracellular polyamine pools invariably inhibits cell growth. This is usually accomplished by inhibiting polyamine biosynthesis. Our previous research demonstrated that the expression of ODC and AdoMetDC was increased in colorectal cancer tissue ${ }^{[11,12]}$, and the downregulation of ODC and AdoMetDC could inhibit colorectal cancer growth ${ }^{[13-15]}$. However, this might be more effectively achieved by the activation of polyamine catabolism at the SSAT level, a strategy first validated in MCF-7 breast carcinoma cells ${ }^{[16]}$. Therefore, in the present study, we constructed a recombinant adenovirus that could express human SSAT and detected its inhibitory effect on colorectal cancer cell growth in vitro.

\section{Materials and methods}

Cell culture and reagents Human colorectal adenocarcinoma cell HT-29 and LoVo were preserved in our laboratory. They were maintained in RPMI-1640 medium supplemented with $10 \%(v / v)$ heat-inactivated bovine serum, $100 \mathrm{U} / \mathrm{mL}$ penicillin, and $100 \mu \mathrm{g} / \mathrm{mL}$ streptomycin at $37^{\circ} \mathrm{C}$ in a humidified atmosphere of $5 \% \mathrm{CO}_{2}$. $\mathrm{HEK} 293$ packaging cells (transformed human embryonic kidney cells) were obtained from the Chinese Academy of Science (Shanghai, China). They were cultured in Dulbecco's modified Eagle's medium (Gibco, Grand Island, NY, USA) containing 10\% fetal bovine serum, penicillin, and streptomycin. The pGL3-hTERT plasmid was a gift from Dr Kou-juey WU (Department of Pathology and Genetics \& Development, Columbia University, New York, USA). The shuttle vector pAdTrack and Escherichia coli $(E$ coli) AdEasy-1 cells (E coli BJ5183 cells transformed with the pAdEasy-1 vector) were purchased from American Type Culture Collection (Manassas, VA, USA). The MTS (3-C4, 5-dimethylthiaol-2-yl)-5-(3-carboxy-methoxyphenyl)2-(-4-sulfophenyl)-2H-tetrazolium, inner salt)/phenazine methosulfate (PMS) kit was purchased from Promega (Madison, WI, USA). The polyamines (spermidine and spermine) were purchased from Sigma (St Louis, MO, USA). The anti-SSAT mouse polyclonal antibody was purchased from Abnova (Taipei, China). The $\beta$-actin antibody was obtained from Santa Cruz Biotechnology (Santa Cruz, CA, USA), and the ECL Western blotting detection system was from Millipore (Bedford, MA, USA).

Amplification of SSAT gene and construction of TA clone The total RNA was extracted from colorectal cancer tissue. Complementary DNA was then synthesized using the cDNA synthesis kit (MBI, Glen Burine, MD, USA). PCR was performed to amplify the SSAT gene. The sequence of the SSAT primers was as follows: forward primer, 5'TAA CCA TGG ATG GCT AAA TTC GTG ATC CGC-3’ and reverse primer, 5'-CGGTCTAGATCACTCCTC TGTTGC CAT TTT-3'. The restriction sites were $\mathrm{NcoI}$ and $\mathrm{XbaI}$ (enzyme recognition sites are underlined). The PCR products were purified using a QIAquick gel extraction kit (Qiagen, Valencia, CA, Germany) and linked to plasmid pMD-18T with a polyA linker. The recombinant was transformed into $E$ coli $\mathrm{DH} 5 \alpha$ and selected by selective culture medium containing ampicillin. The positive recombinant plasmid was identified by dual digestion of restrictive enzymes $N c o$ I and $X b a \mathrm{I}$.

Construction of plasmid pAdTrack-hTERT-SSAT The pMD-18T-SSAT and pGL3-TERT plasmids were digested by restrictive enzymes $N c o$ I and $X b a I$. The digested fragments were collected and purified using a gel extraction kit. The 2 fragments were ligated by $\mathrm{T} 4$ ligase at $16{ }^{\circ} \mathrm{C}$ overnight, and then transformed into $\mathrm{DH} 5 \alpha$ by $\mathrm{CaCl}_{2}$ method. Positive colonies were selected by agar plates containing ampicillin and confirmed by restriction enzyme mapping. The positive recombinant plasmid pGL3-hTERT-SSAT was digested by restrictive enzymes SalI and HindIII, and the small fragment was ligated with pAdTrack, which was also digested by SalI and HindIII. The positive clones (pAdTrack-hTERT-SSAT) were confirmed by digestion.

Construction of pAdEasy-hTERT-SSAT plasmid The pAdTrack-hTERT-SSAT plasmids were digested with PmeI and then purified. In total, $5 \mu \mathrm{g}$ purified linearized plasmid was transformed into AdEasy-1 competent cells prepared by $\mathrm{CaCl}_{2}$ method for the homologous recombinant with pAdEasy-1. The reaction product was then plated onto LB/ kan $(50 \mu \mathrm{g} / \mathrm{mL})$ plates. After $24 \mathrm{~h}$ incubation at $37{ }^{\circ} \mathrm{C}$, approximately 24 of the smallest individual colonies were picked and grown in $5 \mathrm{~mL} \mathrm{LB} / \mathrm{kan}$ for 16-18 h. The plasmids were acquired using a conventional alkaline lysis method. The candidate clones were identified by $P a c$ I digestion and yielded a large (approximately $30 \mathrm{~kb}$ ) and a small fragment $(3.0 \mathrm{~kb}$ or $4.5 \mathrm{~kb}$ ). BamHI was also used to identify the positive clones. The correct recombinant miniprep DNA (pAdEasy-hTERTSSAT) were retransformed into the DH5 $\alpha$ and purified using Qiagen's plasmid midi kit.

Viral packaging and amplification in 293 cells Recombinant adenoviral plasmids (pAdEasy-hTERT-SSAT) were digested with $P a c \mathrm{I}$, ethanol precipitated, and resuspended in sterile $\mathrm{H}_{2} \mathrm{O}$. In total, $30 \mu \mathrm{g}$ of digested pAdEasy-hTERTSSAT plasmids was transfected into 293 packaging cells using Lipofectamine 2000 (Gibco, USA). The cells were cultured for 7-10 $\mathrm{d}$ and harvested. The cells were then frozen at $-80{ }^{\circ} \mathrm{C}$ 
and thawed in a $37{ }^{\circ} \mathrm{C}$ water bath; this procedure was repeated 4 times. The samples were centrifuged at $12000 \times g$ for $10 \mathrm{~min}$, and the viral supernatant was stored at $-80{ }^{\circ} \mathrm{C}$. For further amplification, more 293 cells needed to be infected with these viral stocks. The recombinant virus particles were purified by ultracentrifugation in cesium chloride step gradients. The SSAT gene ligated into the virus DNA was also detected by PCR. The titer of the purified adenovirus was $2 \times 10^{8} \mathrm{pfu} / \mathrm{mL}$.

Analysis of gene transduction efficiency in vitro The efficiency of the adenovirus-mediated gene transfer was assessed by MTS assay. The HT-29 and LoVo cells were seeded at density of 5000 cells/well in 96-well plates and cultured overnight. The cells were then infected with Ad-hTERTSSAT at multiplicities of infection (MOI) of 0, 10, 25, 50, 75, and 100 for $24 \mathrm{~h}$. After $72 \mathrm{~h}$ incubation, $20 \mu \mathrm{L}$ MTS solution (with PMS) was added to each well and maintained for $2 \mathrm{~h}$ at $37^{\circ} \mathrm{C}$. The optical density was measured at $490 \mathrm{~nm}$.

Western blot analysis of SSAT protein After the HT-29 and LoVo cells were treated with phosphate-buffered saline (PBS), Ad-green fluorescent protein (GFP), and Ad-hTERTSSAT by 50 and $25 \mathrm{MOI}$ in 1640 medium containing $5 \%$ fetal bovine serum for $72 \mathrm{~h}$. They were then collected with a cell scraper and washed with ice-cold PBS 3 times. The total cell lysates were prepared in extraction buffer containing 0.05 $\mathrm{mol} / \mathrm{L} \mathrm{Tris}(\mathrm{pH} 8.0$ ), $0.15 \mathrm{~mol} / \mathrm{L} \mathrm{NaCl}, 0.02 \%$ sodium azide, $0.1 \%$ SDS, $100 \mathrm{mg} / \mathrm{mL}$ phenylmethylsulfonyl fluoride, $1 \mathrm{mg} / \mathrm{mL}$ aprotinin, and 1\% NP-40. Sample protein concentrations were qualified by the bicinchoninic acid protein assay. The proteins were subjected to $5 \%$ stacking and $12 \%$ resolving SDS-PAGE gels and transferred to polyvinylidene difluoride membranes (Millipore, USA). After incubation with the appropriate antibodies in PBS containing 5\% non-fat dry milk and $0.02 \%$ Tween-20, the membranes were incubated with a secondary antibody conjugated with horseradish peroxidase. The membranes were then reacted with luminol substrate for $5 \mathrm{~min}$ and exposed to X-ray films (AGFA, Mortsel, Belgium). The results were analyzed using SmartView analysis software (Smarview Enterprise Imaging Solutions, Lake Forest, CA, USA).

Measurement of polyamine content Polyamine content was measured by HPLC analysis. HT-29 and LoVo cells were infected with Ad-GFP and Ad-hTERT-SSAT by 50 and 25 MOI for $3 \mathrm{~d}$ and then harvested by scraping. Intracellular polyamines were extracted from cell pellets with $10 \%$ trichloroacetic acid and then the polyamines were mixed with a 2fold volume of dansyl chloride and dansylated in the presence of sodium carbonate for $20 \mathrm{~min}$ at $70{ }^{\circ} \mathrm{C}$. Dansylated polyamines were quantified using reverse-phase HPLC.
Effect on cell growth To observe the effect of the adenovirus on cell growth in vitro, the MTS assay was used to draw cell growth curves. The cells were inoculated at a density of 4000 cells/well in 96-well plates and cultured overnight. Owing to different infective efficiency, HT-29 and LoVo cells were then treated with PBS, Ad-GFP, and Ad-hTERT-SSAT by 50 and 25 MOI. All experiments were performed 6 times. After 24, 48, 72, 96, and $120 \mathrm{~h}, 20 \mu \mathrm{L}$ MTS solution (with PMS) was added to each well and maintained for $2 \mathrm{~h}$ at $37^{\circ} \mathrm{C}$. The optical density was measured at $490 \mathrm{~nm}$. Cell viability was assessed by absorbance at $490 \mathrm{~nm}$.

Colony-forming assay The HT-29 and LoVo cells were infected with Ad-GFP or Ad-hTERT-SSAT by 50 and 25 MOI. After $24 \mathrm{~h}$, the cells were trypsinized, counted, and seeded for the colony-forming assay on 6-well culture plates $\left(1 \times 10^{3}\right.$ cells/well) in triplicate, respectively. After incubation for $14 \mathrm{~d}$, the colonies were stained with Giemsa. Colonies containing more than 50 cells were scored and the plating efficiency was calculated.

Exogenous polyamine supplementation experiment HT29 and LoVo cells were inoculated at a density of $1 \times 10^{4}$ cells/ well in 6-well plates and cultured overnight. The cells were then infected with Ad-GFP and Ad-hTERT-SSAT by 50 and $25 \mathrm{MOI}$. At the same time, the medium of the infected cells Ad-hTERT-SSAT was supplemented with spermidine (50 $\mu \mathrm{mol} / \mathrm{L})$ or spermine $(25 \mu \mathrm{mol} / \mathrm{L})$, respectively. The medium was changed daily to keep the concentration of the exogenous polyamine concentration stable. After $72 \mathrm{~h}$, the cells were then harvested by trypsinization and stained with $0.4 \%$ trypan blue (Gibco, USA) to reveal the dead cells. Viable cells were then counted with a hemocytometer.

Statistical analysis Data are presented as the mean \pm SD from 3 separate experiments. Student's $t$-test was used to compare the data and $P<0.05$ was taken as the level of significance. All results were analyzed using the SPSS statistical software package (Chicago, IL, USA).

\section{Results}

Identification of adenoviral backbone DNA pAdEasyhTERT-SSAT The SSAT gene was amplified by RT-PCR. The PCR products were separated by electrophoresis and an approximately 516 bp band was obtained, which is consistent with the size of the SSAT gene (gi: 4506788, Figure 1A). A fragment approximately $500 \mathrm{bp}$ was also obtained after the constructed TA vector was digested (Figure 1A). It demonstrated that the PCR product was successfully inserted into pMD-18T.

To identify whether the SSAT gene had been inserted into the plasmid pGL3-hTERT, the constructed plasmid pGL3- 
A

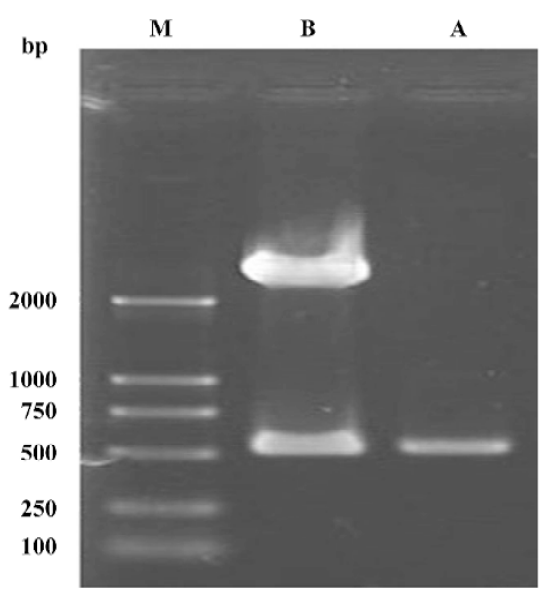

C

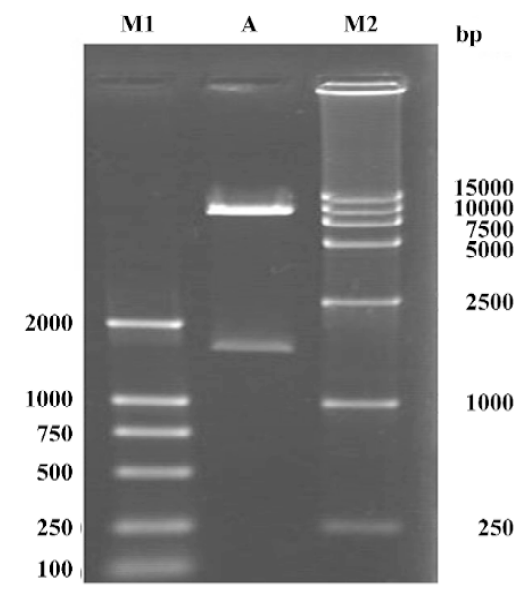

B

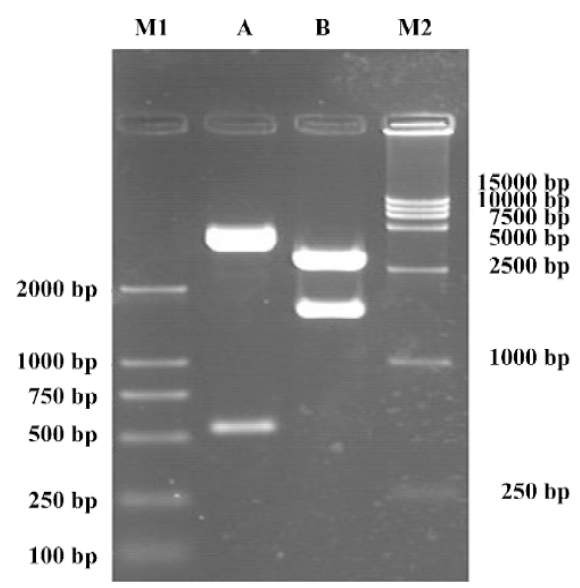

D

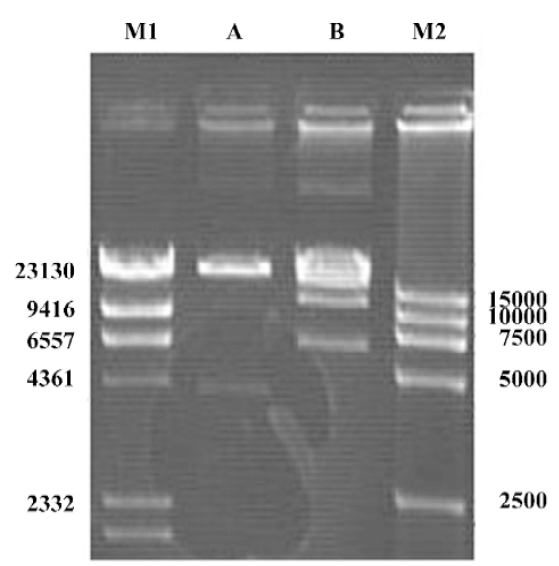

Figure 1. Restrictive digestion maps in the construction of pAdEasy-hTERT-SSAT. (A) RT-PCR product and digestion of pMD18T-SSAT construction. Lane M: DL2000 marker; lane A: SSAT RT-PCR product; lane B: pMD18T-SSAT plasmid digested by NcoI and XbaI. (B) digestion of pGL3-hTERT-SSAT plasmid. Lane M1: DL2000 marker; lane M2: DL15000 marker; lane A: pGL3-hTERT-SSAT plasmid digested by $\mathrm{NcoI}$ and $\mathrm{XbaI}$. (516 bp, $4.5 \mathrm{~kb}$ ); lane B: pGL3-hTERT-SSAT plasmid digested by Sall and HindIII (1.5 kb, $3.5 \mathrm{~kb})$. (C) digestion of pAdTrack-hTERT-SSAT plasmid. Lane M1: DL2000 marker; lane M2: DL15000 marker; lane A: pAdTrack-hTERT-SSAT plasmid digested by SalI and HindIII $(1.5 \mathrm{~kb}, 8.3 \mathrm{~kb})$. (D) restriction digestion of pAdEasy-hTERT-SSAT. Lane M1: $\lambda$-HindIII digested marker; lane M2: DL-15000 marker; lane A: digestion of pAdEasy-hTERT-SSAT by PacI (4.5 kb, $35 \mathrm{~kb})$; lane B: digestion of pAdEasy-hTERT-SSAT by BamHI $(7 \mathrm{~kb}, 11.7 \mathrm{~kb}, 21.7 \mathrm{~kb})$.

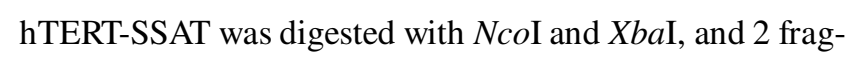
ments ( $516 \mathrm{bp}$ and $4.5 \mathrm{~kb}$ ) were found in $1 \%$ agarose gel electrophoresis (Figure 1B). The pGL3-hTERT-SSAT construct was also digested by SalI and HindIII, and 2 fragments ( $1.5 \mathrm{~kb}$ and $3.5 \mathrm{~kb}$ ) were observed (Figure 1B). The pAdTrack-hTERT-SSAT plasmids were digested by SalI and HindIII to identify whether the fragment (-hTERT-SSAT-) was inserted, and 2 fragments $(1.5 \mathrm{~kb}$ and $8.3 \mathrm{~kb})$ were found (Figure 1C). These results suggested that the shuttle plasmid pAdTrack-hTERT-SSAT was successfully constructed.
The recombinant shuttle vector was transformed into $E$ coli AdEasy-1 cells for homologous recombination with the pAdEasy-1 vector. Candidate clones were digested with $P a c \mathrm{I}$ or BamHI to identify proper recombination. With PacI digestion, 2 fragments $(4.5 \mathrm{~kb}$ and $35 \mathrm{~kb})$ were produced (Figure 1D). With Bam HI, a $7 \mathrm{~kb}$ fragment was produced in addition to the 11.7 and $21.7 \mathrm{~kb}$ fragments generated from the pAdEasy-1 sequences (Figure 1D).

Production of the adenovirus in $\mathbf{2 9 3}$ cells In order to package the viruses, $30 \mu \mathrm{g}$ of digested pAdEasy-hTERT- 
SSAT plasmids was transfected into 293 packaging cells. The viral production process was monitored by GFP expression. GFP expression could be observed $24 \mathrm{~h}$ after transfection (Figure 2A), which indicated that the virus particles had been packaged. The viruses were identified by PCR with the supernatant fluid using the SSAT primers, and an approximately 516 bp band was obtained (Figure 3 ). To acquire more viruses, the generated viral particles were used to infect more 293 cells, and a stronger GFP expression was observed (Figure 2B).
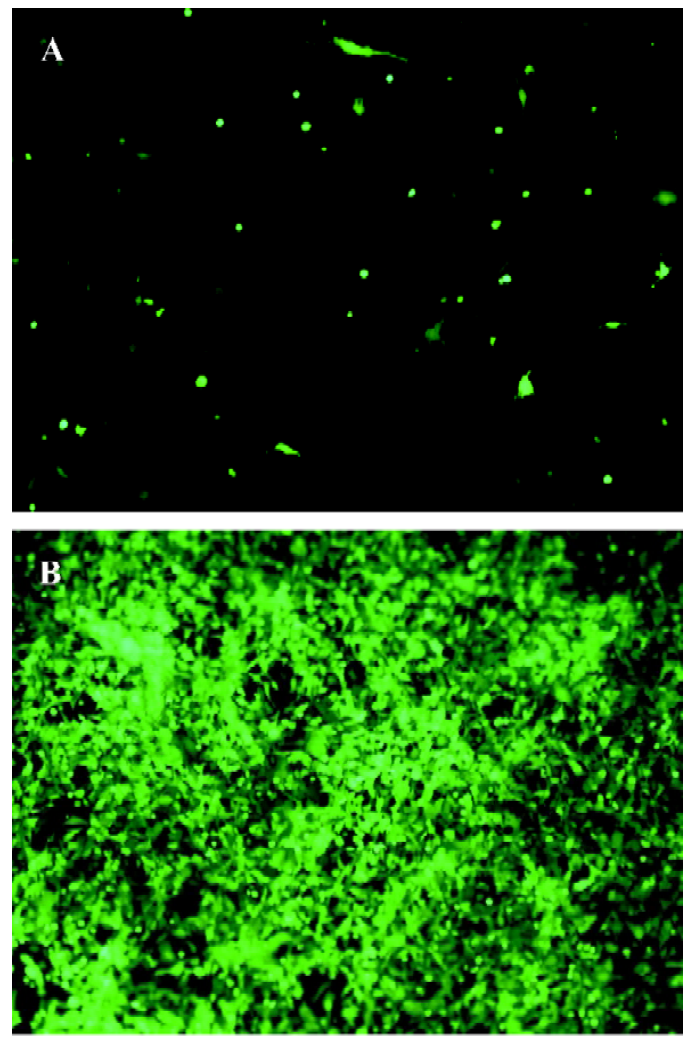

Figure 2. Fluorescence micrographs of GFP expression in 293 cells. (A) GFP expression in 293 cells $24 \mathrm{~h}$ after transfection with pAdEasyhTERT-SSAT; (B) GFP expression in 293 cells $48 \mathrm{~h}$ after infection with first-generation adenovirus.

Gene transduction efficiency in vitro The results (Figure 4A, 4B) showed that there was dose-dependent growth inhibition in both HT-29 and LoVo cell lines. It reflected the transduction efficiency of the adenovirus in different cell lines of colorectal cancer cells. The LoVo cell line was more sensitive to infection of Ad-hTERT-SSAT. Owing to the differential sensitivities of various cell lines, we chose 50 and 25 MOI of the adenovirus to infect HT-29 and LoVo cells. Under these conditions, Ad-hTERT-SSAT was more suppressive of growth than the control Ad-GFP virus, while Ad-GFP

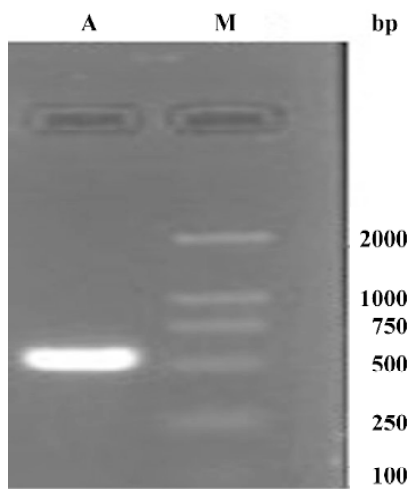

Figure 3. PCR product with the supernatant fluid. Lane M: DL2000 marker; lane A: PCR product (516 bp).
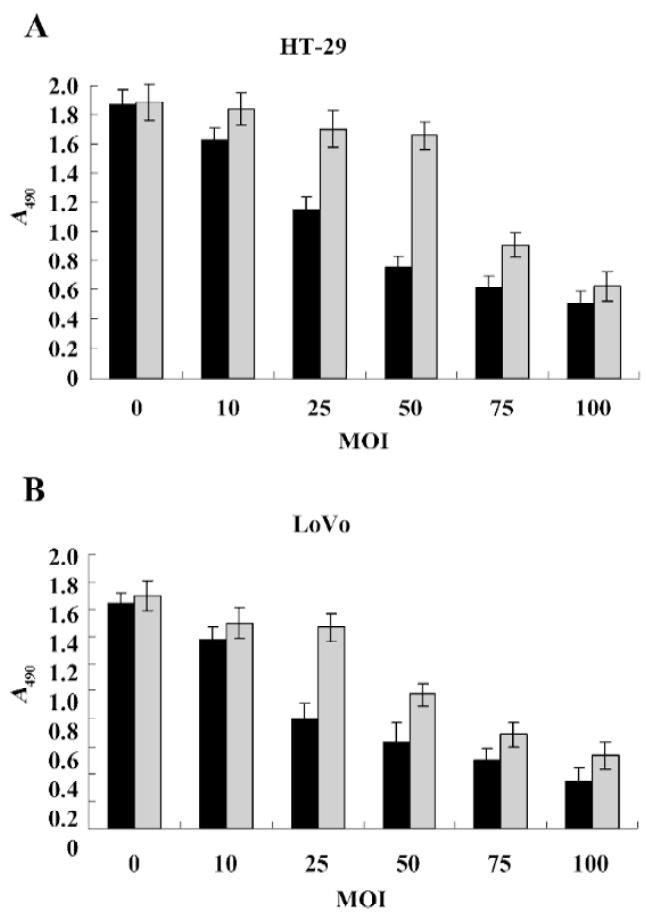

Figure 4. Effect of Ad-hTERT-SSAT at various titers on growth of HT-29 (A) and LoVo cells (B). Both cells were infected with either Ad-hTERT-SSAT (匹) or Ad-GFP (匹) at 0-100 MOI doses.

had no obviously toxic effects on the cells.

Effect of Ad-hTERT-SSAT on SSAT gene expression in HT-29 cells To detect the effect of replication-deficient AdhTERT-SSAT infection on intracellular SSAT protein levels, Western blot analysis was performed and the results showed that SSAT protein levels were significantly increased in AdhTERT-SSAT-treated HT-29 cells when compared with AdGFP or PBS-treated cells (Figure 5).

Inhibitory effect of Ad-hTERT-SSAT on polyamine content in colorectal cancer cells After proving that Ad-hTERT- 
A

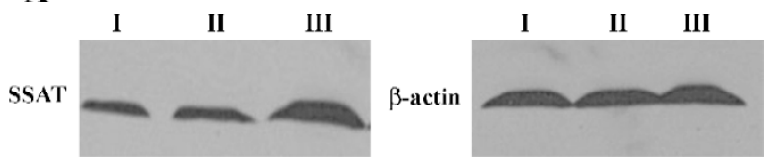

B

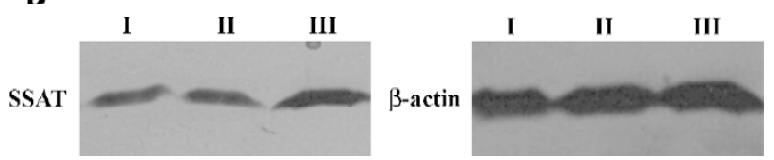

Figure 5. Western blot analysis for SSAT expression in HT-29 (A) and LoVo cells (B). HT-29 and LoVo cells were infected with adenoviruses by 50 and 25 MOI. I, no virus-treated cells; II, Ad-GFP-infected cells; III, Ad-hTERT-SSAT-infected cells.

SSAT could increase SSAT protein expression in colorectal cancer cells, we next evaluated whether the polyamine concentration decreased accordingly. Polyamines in adenovirusinfected or -uninfected colorectal cancer cells were separated by ion-pairing, reverse-phase HPLC. As shown in Table 1, Ad-hTERT-SSAT decreased the content of spermidine and spermine in HT-29 and LoVo cells, which was correlated with the acceleration of polyamine catabolism.

Table 1. Effect of Ad-hTERT-SSAT on polyamine levels in HT-29 and LoVo human colon cancer cells. $n=3$. Data are mean \pm SD. ${ }^{\mathrm{b}} P<0.05$ $v s$ infection with Ad-GFP or uninfected cells.

\begin{tabular}{lccc}
\hline & \multicolumn{3}{c}{ Polyamine pools (pmol/10 $0^{6}$ cells) } \\
Cell lines and treatment & Putrescine & Spermidine & Spermine \\
\cline { 2 - 4 } & & & \\
\hline & $241 \pm 30$ & $10317 \pm 1091$ & $12156 \pm 1437$ \\
HT-29 & $253 \pm 26$ & $9783 \pm 1021$ & $11032 \pm 1275$ \\
+Ad-GFP & $279 \pm 33$ & $5172 \pm 659^{\mathrm{b}}$ & $6354 \pm 721^{\mathrm{b}}$ \\
+ Ad-hTERT-SSAT & $293 \pm 37$ & $6135 \pm 632$ & $7928 \pm 833$ \\
LoVo & $331 \pm 39$ & $5987 \pm 653$ & $7237 \pm 769$ \\
+Ad-GFP & $363 \pm 42$ & $2035 \pm 399^{\mathrm{b}}$ & $2758 \pm 328^{\mathrm{b}}$ \\
+ +Ad-hTERT-SSAT & & & \\
\hline
\end{tabular}

Inhibitory effect of Ad-hTERT-SSAT on colorectal cancer cells We examined the inhibitory effects of Ad-hTERTSSAT on the growth of HT-29 and LoVo cells in vitro using the MTS assay and drew the cell growth curves (Figure 6A, 6B). The expression of SSAT affected the growth of both HT-29 and LoVo cells. Ad-hTERT-SSAT inhibited their proliferation when compared with the control groups (treated with Ad-GFP or PBS).

In the colony-forming assay, plating efficiencies in HT29, Ad-GFP-infected HT-29, and Ad-hTERT-SSAT-infected HT-29 were $65.5 \% \pm 8.0 \%, 60.4 \% \pm 5.6 \%$, and $8.5 \% \pm 3.2 \%$,
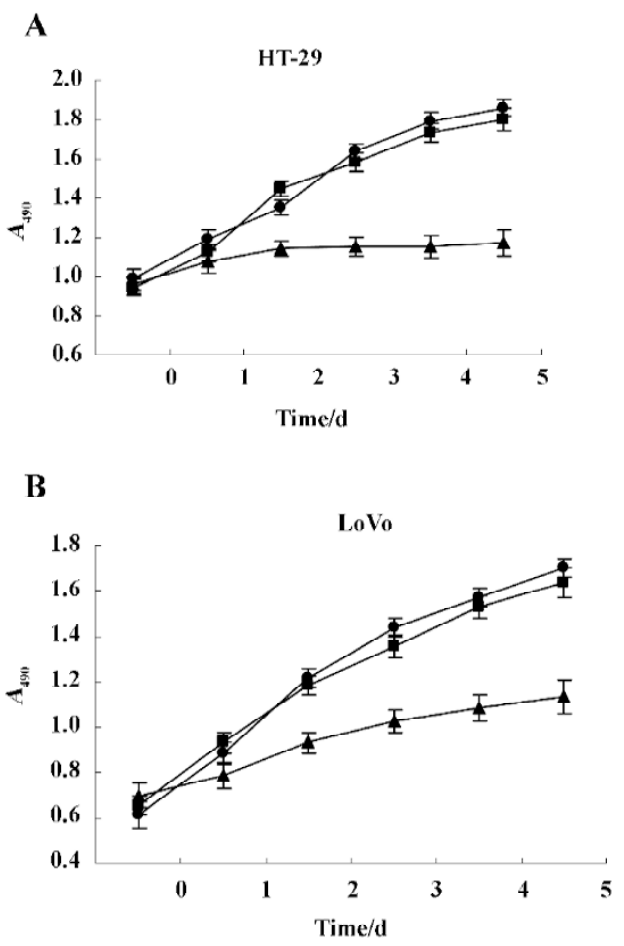

Figure 6. Effect of Ad-hTERT-SSAT on growth of HT-29 (A) and LoVo (B) cells. HT-29 and LoVo cells were infected with either AdhTERT-SSAT( $\mathbf{A})$ or Ad-GFP (a) at 50 and 25 MOI doses. After $24 \mathrm{~h}$ incubation, absorbance was measured. Growth curves of Ad-hTERTSSAT-infected cells ( $\mathbf{\Delta}$ ), Ad-GFP-infected cells ( $\bullet$ ), and no virustreated cells $(\bullet)$. Absorbance was measured every day for a period of $5 \mathrm{~d}$.

respectively; Plating efficiencies in LoVo, Ad-GFP-infected LoVo, and Ad-hTERT-SSAT-infected LoVo were $62.5 \% \pm 9.6 \%$, $58.7 \% \pm 8.6 \%$, and $30.5 \% \pm 5.4 \%$, respectively (Figure 7). The plating efficiency in Ad-hTERT-SSAT-infected cells was significantly decreased compared with their 2 controls $(P<0.05)$.

To further examine whether the antiproliferative effect of Ad-hTERT-SSAT can be antagonized by exogenous polyamines, spermidine and spermine were added to restore HT-29 and LoVo cell proliferation. The growth arrest caused by AdhTERT-SSAT was partially reversed by spermidine and spermine (Table 1).

\section{Discussion}

There are many biochemical alterations in colorectal cancer cells, but one of the most consistent changes is the elevation of the intracellular polyamine content ${ }^{[17]}$. The depletion of intracellular polyamine pools invariably inhibits cell growth. Although this is usually accomplished by inhibiting polyamine biosynthesis, this might be more effectively achieved by the activation of polyamine catabolism at the SSAT level, a strat- 

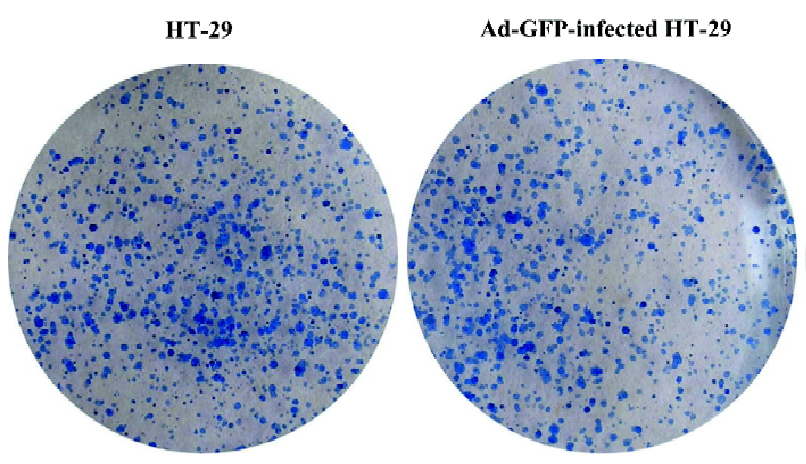

Ad-hTERT-SSAT-infected HT-29
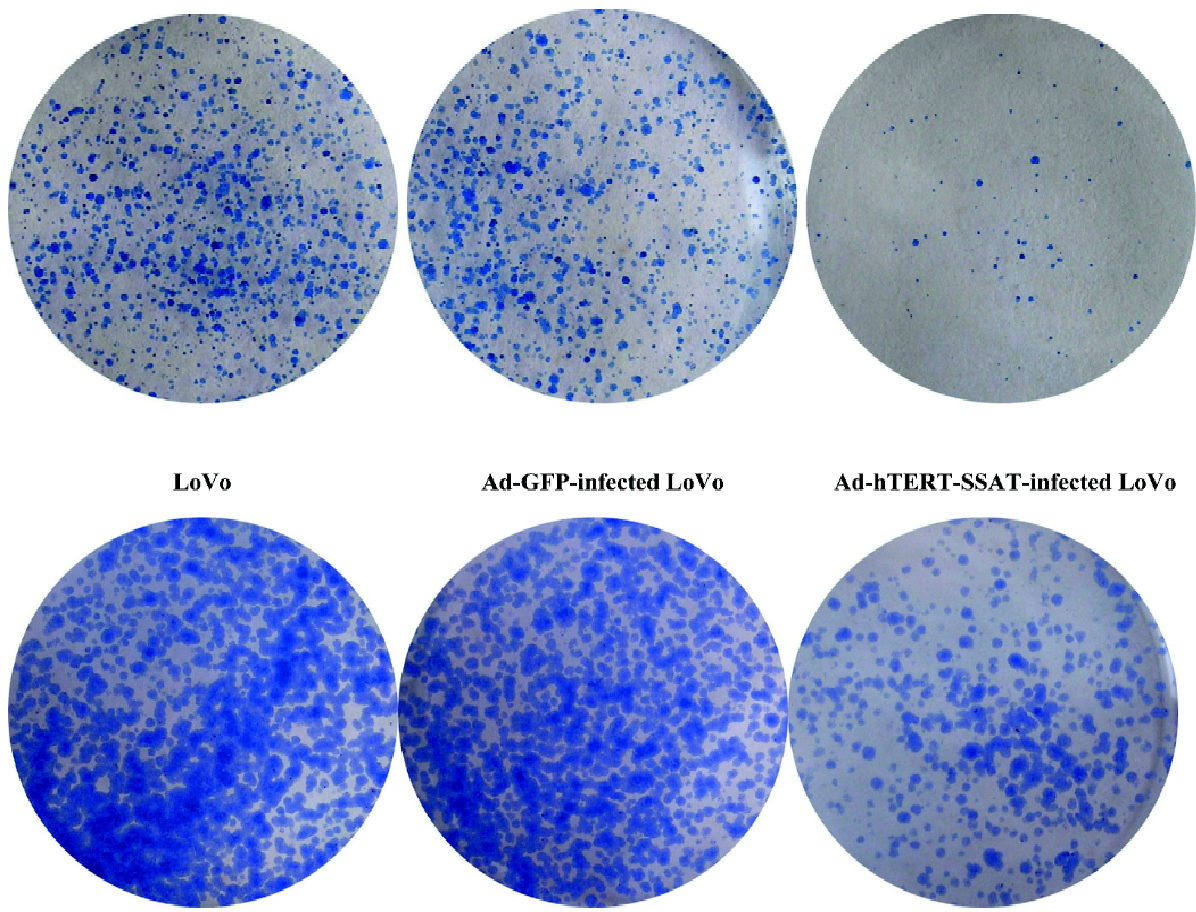

Ad-hTERT-SSAT-infected LoVo

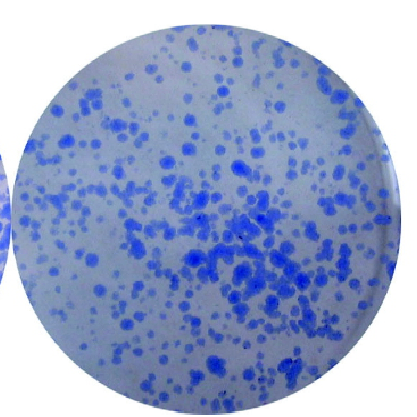

Figure 7. Effect of Ad-hTERT-SSAT on growth of HT-29 and LoVo cells. Cells were cultured in triplicate sets of 6-well tissue culture plates with $1640 \%+10 \%$ NBS for $14 \mathrm{~d}$. Colonies were visualized by Giemsa staining. Colonies in Ad-hTERT-SSAT-infected cells were significantly decreased compared to their 2 controls $(P<0.05)$.

Table 2. Polyamine supplementation on Ad-hTERT-SSAT-induced growth suppression in HT-29 and LoVo cells. $n=3$. Data are mean \pm SD. ${ }^{\mathrm{b}} P<0.05$ vs Ad-hTERT-SSAT-treated cells.

\begin{tabular}{|c|c|c|}
\hline \multirow[t]{2}{*}{ Treatment } & \multicolumn{2}{|c|}{ Cell number ( $\%$ of control) } \\
\hline & HT-29 cells & LoVo cells \\
\hline Ad-GFP & $100 \pm 12.5$ & $100 \pm 10.6$ \\
\hline Ad-hTERT-SSAT & $34 \pm 4.3$ & $42 \pm 3.5$ \\
\hline Ad-hTERT-SSAT+spermidine & $71 \pm 3.1^{\mathrm{b}}$ & $70 \pm 4.2^{\mathrm{b}}$ \\
\hline Ad-hTERT-SSAT+spermine & $66 \pm 2.8^{\mathrm{b}}$ & $67 \pm 3.0^{\mathrm{b}}$ \\
\hline
\end{tabular}

egy first validated in MCF-7 breast carcinoma cells ${ }^{[16]}$. Therefore, the polyamine catabolism pathway may become another potent and attractive target for cancer therapy.

SSAT is the principle catabolic enzyme responsible for the regulation of the intercellular polyamine content in mammalian cells. SSAT transfers an acetyl group from acetylCoA to the $\mathrm{N}^{1}$ positions of spermidine and spermine. The corresponding $\mathrm{N}^{1}$-acetyl derivatives are either excreted outwards or undergo further metabolism by polyamine oxidase, ultimately yielding putrescine, which is a pathway opposite to that of polyamine synthesis. SSAT is thought to play a key role in maintaining a properly balanced ratio of polyamine in cells and in preventing the over accumulation of higher polyamines that may become cytotoxic ${ }^{[17,19]}$. Whereas SSAT activity is normally very low in cells and tissues, the enzyme is strongly induced by elevations in intracellular polyamines and in response to terminally-alkylated polyamine analogs that are currently undergoing clinical trials as cancer chemotherapeutic agents ${ }^{[20,21]}$. A number of independent lines of evidence suggest that non-steroidal anti-inflammatory drugs (NSAIDs) can prevent colorectal cancer. Martin et al found that aspirin was able to induce SSAT expression and decrease intracellular polyamine levels in HT-29 colon adenocarcinoma cells ${ }^{[22]}$. They also demonstrated that the induction of polyamine catabolism leading to the reduction in intracellular polyamines could be one of the general mechanisms for the chemopreventive actions of different NSAIDs in colon cancer ${ }^{[23]}$.

There are a number of viral and non-viral delivery routes and methods for gene transfer used in gene therapy ${ }^{[24-27]}$. Due to its unparalleled capacity for gene transfer, stability, high titers, and low risk of mutagenesis ${ }^{[28]}$, adenovirus vectors were chosen in our study to transfer the SSAT gene into colorectal cancer. 
In the present study, we successfully constructed a replication-deficient recombinant adenovirus that can simultaneously express the SSAT gene. Western blot analysis demonstrated that Ad-hTERT-SSAT significantly increased SSAT protein levels in HT-29 and LoVo cells. Furthermore, with a substantial increase in SSAT expression, spermidine and spermine decreased to low levels after Ad-hTERT-SSAT infection. In addition, our data showed that Ad-hTERT-SSAT had a significant inhibitory effect on colorectal cancer cell growth. Polyamines are known to play a key role in maintaining a high cell proliferation rate. Areduction in polyamines may contribute to the suppression of cancer growth. The cell growth inhibition due to Ad-hTERT-SSAT infection can be partially reversed by exogenous polyamines.

In summary, the experimental data presented here demonstrate that the adenovirus-mediated expression of SSAT induces polyamine catabolism and leads to the significant suppression of colorectal cancer cell growth. Although many details must be elucidated in its antitumor effect, adenoviral vector-mediated SSAT provides a novel treatment option for colorectal cancer.

\section{References}

1 Porter CW, Regenass U, Bergeron RJ. In: Dowling RH, Folsch UR, Loser C, editors. Falk symposium on polyamines in the gastrointestinal tract; Dordrecht, Netherlands: Kluwer Academic Publishers Group; 1992. p301-22.

2 Auvinen M, Paasinen A, Andersson LC, Holtta E. Ornithine decarboxylase activity is critical for cell transformation. Nature 1992; 360: 355-8.

3 Moshier JA, Dosescu J, Skunca M, Luk GD. Transformation of NIH/3T3 cells by ornithine decarboxylase overexpression. Cancer Res 1993; 53: 2618-22.

4 Pegg AE, Xiong H, Feith DJ, Shantz LM. S-adenosylmethionine decarboxylase: structure, function and regulation by polyamines. Biochem Soc Trans 1998; 26: 580-6.

5 Marton LJ, Pegg AE. Polyamines as targets for therapeutic intervention. Annu Rev Pharmacol Toxicol 1995; 35: 55-91.

6 Glikman P, Vegh I, Pollina MA, Mosto AH, Levy CM. Ornithine decarboxylase activity, prolactin blood levels, and estradiol and progesterone receptors in human breast cancer. Cancer 1987; 60: 2237-43.

7 Upp JR, Saydjari R, Townsend CM, Singh P, Barranco SC, Thompson JC. Polyamine levels and gastrin receptors in colon cancers. Ann Surg 1988; 207: 662-9.

8 Gerner EW, Meyskens FL. Polyamines and cancer: old molecules, new understanding. Nat Rev Cancer 2004; 4: 781-92.

9 Loser C, Folsch UR, Paprotny C, Creutzfeldt W. Polyamines in colorectal cancer. Evaluation of polyamine concentrations in the colon tissue, serum and urine of 50 patients with colorectal cancer. Cancer 1990; 65: 958-66.

10 Higuchi CM, Wang W. Comodulation of cellular polyamines and proliferation: biomarker application to colorectal mucosa. J
Cell Biochem 1995; 57: 256-61

$11 \mathrm{Hu}$ HY, Liu XX, Jiang CY, Zhang Y, Bian JF, Lu Y, et al. Cloning and expression of ornithine decarboxylase gene from human colorectal carcinoma. World J Gastroenterol 2003; 9: 714-6.

$12 \mathrm{Hu}$ HY, Liu XX, Jiang CY, Lu Y, Liu SL, Bian JF, et al. Ornithine decarboxylase gene is overexpressed in colorectal carcinoma. World J Gastroenterol 2005; 11: 2244-8.

13 Zhang B, Liu XX, Zhang Y, Jiang CY, Teng QS, Hu HY, et al. Adenovirus-mediated expression of both antisense ODC and AdoMetDC inhibits colorectal cancer cell growth in vitro. Acta Pharmacol Sin 2006; 27: 353-9.

14 Zhang B, Liu XX, Zhang Y, Jiang CY, Hu HY, Gong L, et al. Polyamine depletion by ODC-AdoMetDC antisense adenovirus impairs human colorectal cancer growth and invasion in vitro and in vivo. J Gene Med 2006; 8: 980-9.

15 Chi WL, Song XR, Jiang CY, Liu XX, Li WT, Wang XW. Lentiviral vector-mediated downregulation of ornithine decarboxylase inhibits tumor cell growth in vitro and in vivo. Tumor Biol 2006; 27: 243-51.

16 Vujcic S, Halmekyto M, Diegelman P, Gan G, Kramer DL, Janne $\mathrm{J}$, et al. Effects of conditional overexpression of spermidine/ spermine $N^{1}$-acetyltransferase on polyamine pool dynamics, cell growth, and sensitivity to polyamine analogs. J Biol Chem 2000; 275: 38 319-28.

17 Wallace HM, Caslake R. Polyamines and colon cancer. Eur J Gastroenterol Hepatol 2001; 13: 1033-9.

18 Casero RA, Pegg AE. Spermidine/spermine $\mathrm{N}^{1}$-acetyltransferase: the turning point in polyamine metabolism. FASEM J 1993; 7 : 653-61.

19 Seiler N. Functions of polyamine acetylation. Can J Physiol Pharmacol 1987; 65: 2024-35.

20 Marton LJ, Pegg AE. Polyamines as targets for therapeutic intervention. Annu Rev Pharm 1995; 35: 55-91.

21 Casero RA, Woster PM. Terminally alkylated polyamine analogues as chemotherapeutic agents. J Med Chem 2001; 44: 1-26.

22 Martin ME, O’Brien TG, Fultz KE, Babbar N, Yerushalmi H, Qu $\mathrm{N}$, et al. Pronounced reduction in adenoma recurrence associated with aspirin use and a polymorphism in the ornithine decarboxylase gene. Proc Natl Acad Sci USA 2003; 100: 7859-64,

23 Babbar N, Gerner EW, Casero RA. Induction of spermidine/ spermine $\mathrm{N}^{1}$-acetyltransferase (SSAT) by aspirin in Caco-2 colon cancer cells. Biochem J 2006; 394: 317-24.

24 Yi C, Huang Y, Guo ZY, Wang SR. Antitumor effect of cytosine deaminase/5-fluorocytosine suicide gene therapy system mediated by Bifidobacterium infantis on melanoma. Acta Pharmacol Sin 2005; 26: 629-34.

25 Zhang SN, Yuan SZ, Zhu ZH, Wen ZF, Huang ZQ, Zeng ZY. Apoptosis induced by 5 -flucytosine in human pancreatic cancer cells genetically modified to express cytosine deaminase. Acta Pharmacol Sin 2000; 21: 655-9.

$26 \mathrm{Hu}$ YC. Baculovirus as a highly efficient expression vector in insect and mammalian cells. Acta Pharmacol Sin 2005; 26: 405-16.

$27 \mathrm{Li} \mathrm{L}, \mathrm{He}$ DL. Transfection of promyelocytic leukemia in retrovirus vector inhibits growth of human bladder cancer cells. Acta Pharmacol Sin 2005; 26: 610-5.

28 Kanerva A, Hemminki A. Modified adenoviruses for cancer gene therapy. Int J Cancer 2004; 110: 475-80. 